\title{
Direct Current Cardioversion in Atrial Fibrillation Patients on Edoxaban Therapy Versus Vitamin K Antagonists: a Real-world Propensity Score-Matched Study
}

\author{
Anna Rago ${ }^{1} \cdot$ Andrea Antonio Papa $^{1}$ (1) - Emilio Attena ${ }^{2} \cdot$ Valentina Parisi $^{3} \cdot$ Paolo Golino $^{1}$ • \\ Gerardo Nigro $^{1} \cdot$ Vincenzo Russo $^{1}$
}

Accepted: 10 September 2020 / Published online: 18 September 2020

(C) The Author(s) 2020

\begin{abstract}
Purpose The purpose of the present study was to compare the long-term effectiveness and safety of newly initiated anticoagulation with edoxaban (EDO) versus uninterrupted vitamin K antagonist (VKA) therapy in patients with atrial fibrillation (AF) scheduled for transesophageal echocardiogram (TEE)-guided direct electrical current cardioversion (DCC).

Methods A propensity score-matched cohort observational study was performed comparing the safety and effectiveness of edoxaban versus well-controlled VKA therapy among a cohort of consecutive non-valvular AF patients scheduled for DCC. The primary safety outcome was major bleeding. The primary efficacy outcome was the composite of stroke, transient ischemic attack (TIA), and systemic embolism (SE).

Findings A total of $130 \mathrm{AF}$ patients receiving edoxaban 60-mg (EDO) treatment were compared with the same number of VKA recipients. The cumulative incidence of major bleedings was $1.54 \%$ in the EDO group and 3.08\% in the VKA group $(P=0.4)$. The cumulative incidence of thromboembolic events was $1.54 \%$ in the EDO group and $2.31 \%$ in the VKA group $(P=0.9)$. A non-significant trend in improved adherence was observed between the EDO and VKA groups with a total anticoagulant therapy discontinuation rate of $4.62 \%(6 / 130)$ vs $6.15 \%(8 / 130)$, respectively $(P=0.06)$.

Implications Our study provides the evidence of a safe and effective use of edoxaban in this clinical setting, justified by no significant difference in major bleedings and thromboembolic events between edoxaban and well-controlled VKA treatments.
\end{abstract}

Keywords Atrial fibrillation - Transesophageal echocardiogram · Direct electrical current cardioversion - Edoxaban . Uninterrupted vitamin K antagonists

\section{Introduction}

Cardioversion (both electric and pharmacological) in AF patients is associated with an increased risk of thromboembolic events $[1,2]$ and an adequate level of periprocedural anticoagulation is essential to reduce this risk. The use of direct oral anticoagulants (DOACs) in clinical practice is rapidly

Andrea Antonio Papa

andreaantoniopapa@libero.it

1 University of the Study of Campania "Luigi Vanvitelli" - Monaldi Hospital, P.zzale E. Ruggeri, 80131 Naples, Italy

2 Department of Cardiology, Health Authority Naples, Naples, Italy

3 Department of Translational Medical Sciences, University of Naples Federico II, Naples, Italy increasing [3-9], even in the setting of patients with AF undergoing electrical cardioversion [10-13], and the current guidelines recommend initiating anticoagulation with DOACs as soon as possible before every cardioversion of AF [14, 15]. For patients with $\mathrm{AF}$ of $>48$-h duration, the current recommendation is to start oral anticoagulation at least 3 weeks before cardioversion and continue it for at least 4 weeks afterwards. Edoxaban (EDO), a direct oral factor Xa inhibitor, was demonstrated to be non-inferior to enoxaparin-warfarin therapy in terms of composite net clinical benefit outcome of stroke, systemic embolic event, transient ischemic attack, myocardial infarction, cardiovascular mortality, and major bleeding events in patients undergoing cardioversion of atrial fibrillation [16]. However, there are no real-world data on clinical outcomes following direct current cardioversion (DCC) in AF patients treated with edoxaban. The aim of the present study was to investigate the safety and effectiveness of newly initiated 
anticoagulation with edoxaban versus uninterrupted vitamin $K$ antagonists (VKA) therapy in patients with non-valvular AF scheduled for transesophageal echocardiography (TEE)-guided DCC.

\section{Materials and Methods}

\section{Database}

Atrial Fibrillation Research Database (NCT03760874) is a prospectively maintained database shared by three cardiologic centers in Italy (Monaldi Hospital, Naples; University of Campania "Luigi Vanvitelli", Naples; Departement of Cardiology, Health Authority Naples) which includes all patients with non-valvular AF followed at our institution between January 2017 and January 2019. At each 6-month follow-up visit, the clinical status, occurrence of stroke, transient ischemic attack (TIA), systemic embolism (SE), myocardial infarction, major bleeding (MB), clinically relevant non-major bleedings (CRNMB), or other side effects were assessed. Informed consent was obtained from all participants before inclusion in the database. The database and the present analysis were approved by the local institutional review committee.

\section{Definitions}

Ischemic stroke was defined as an episode of neurologic deficit lasting $>24 \mathrm{~h}$ in the absence of an intracranial hemorrhage and diagnosed by cerebral computerized tomography. TIA was defined as a temporary neurologic deficit lasting $<24 \mathrm{~h}$. SE was defined as an acute vascular occlusion of an extremity or organ. MB was defined as that which was clinically overt and associated with any of the following: fatal outcome, involvement of a critical anatomic site, fall in hemoglobin $\geq 2 \mathrm{~g} / \mathrm{dL}$, transfusion of $>2 \mathrm{U}$ of whole blood, or packed red blood cells, according to the International Society on Thrombosis and Hemostasis (ISTH) criteria [17]. CRNMB was defined as overt bleeding not meeting the criteria for $\mathrm{MB}$ but requiring medical intervention, unscheduled contact (visit or telephone) with a physician, temporary interruption of study drug (i.e., delayed dosing), pain, or impairment of daily activities according to ISTH criteria [17]. Well-controlled VKA therapy was defined as VKA treatment with high time in therapeutic range $(>70 \%)$.

\section{Patient Population}

The database was queried for patients with persistent AF who underwent TEE-guided DCC during the time period from January 2017 to January 2019 who received edoxaban or VKA treatment. Patients with a follow-up $\leq 360$ days from the first qualifying anticoagulant prescription and VKA with time in therapeutic range $<70 \%$ have been excluded from the analysis, in order to perform an accurate analysis of long-term safety and effectiveness of optimal anticoagulant therapy in real-world AF patients. Propensity score matching analysis generated two groups (edoxaban vs VKA) with minimal differences in baseline characteristics.

\section{Outcomes}

The occurrence of MB events, including the intracranial hemorrhage (ICH), was the primary safety outcome. Occurrence of TE was the primary effectiveness outcome. The secondary effectiveness outcome included death from any cause; the secondary safety outcome included CRNMB events.

\section{Statistical Analysis}

The Kolmogorov-Smirnov normality test was used to analyze data normality. Continuous variables were reported using the mean and standard deviation. Categorical variables were indicated as frequency counts and percentages. Baseline characteristics between edoxaban and VKA groups were compared by $t$ test for continuous variables and Chi-squared test for categorical variables. Nearest neighbor propensity score matching with 1:1 ratio was used to minimize bias between VKA and edoxaban groups. The variables included in the propensity score were age, female sex, BMI, hypertension, $\mathrm{CHA}_{2} \mathrm{DS}_{2}$-VASc score, HAS-BLED score, diabetes mellitus, heart failure, prior stroke/TIA, prior myocardial infarction (MI), glomerular filtration rate, left atrial diameter, indexed left atrial volume, and left ventricle ejection fraction. The incidence of thromboembolic and bleeding events was calculated both as incidence rate every 100 patient-years and as cumulative incidence. We considered as statistically significant all $P$ values less than 5\%. All statistical analyses were performed using Rstudio (RStudio Team (2016). RStudio: Integrated Development for R. RStudio, Inc., Boston, MA URL http://www.rstudio.com/).

\section{Results}

We identified 495 consecutive patients with persistent AF scheduled for TEE-guided DCC during the time period from January 2017 to January 2019 who received edoxaban $(n=$ $230)$ or VKA treatment $(n=210)$. We excluded patients with a follow-up $\leq 360$ days from the first qualifying anticoagulant prescription $(n=20)$ and VKA patients with time in therapeutic range $<70 \%(n=35)$.

Propensity score matching identified 130 edoxaban and the same number of VKA recipients scheduled for DCC who were comparable with respect to demographic and clinical characteristics. Baseline characteristics of the study 
population before and after propensity score matching are summarized in Table 1. The mean follow-up was $20 \pm$ 4 months. Among overall VKA patients before propensity score matching $(n=210)$, the INR tests were performed every 10 days; therefore, about 12,600 INR tests were overall performed during the observational period.

The TEE revealed the presence of a left atrial appendage thrombus in two patients. These patients showed high thromboembolic risk and moderate renal impairment: one patient $(0.7 \%)$ in the edoxaban group (30 mg OD; CHA2DS2VASc score: 5 ; and glomerular filtration rate (GFR): $38 \mathrm{~mL} / \mathrm{min}$ ) and one patient $(0.7 \%)$ in the VKA group (INR: 2.1 ; CHA2DS2VASc score: 4 and GFR: $35 \mathrm{~mL} / \mathrm{min})$. The DCC was performed within the target time range of $23 \pm 2$ days. The acute cardioversion success rate was $86.9 \%(n=113$ / $130)$ in the edoxaban group and $83.8 \%(n=109 / 130)$ in the VKA group $(P=0.4)$. Four weeks after DCC, all patients continued taking anticoagulant therapy due to having a CHA2DS2VASc score $\geq 2$.

A total of six patients among study population (two in EDO and four in VKA) experienced primary safety outcome. The cumulative incidence of major bleedings was $1.54 \%$ in the EDO group and $3.08 \%$ in the VKA group $(P=0.4)$. A total of five patients among study population (two in EDO and three in VKA) experienced primary effectiveness outcome. The cumulative incidence of thromboembolic events was $1.54 \%$ in the EDO group and $2.31 \%$ in the VKA group
$(P=0.9)$. A total of three patients died among study population (one in EDO and two in VKA). The cumulative incidence of all-cause mortality was $0.77 \%$ in the EDO group and $1.54 \%$ in the VKA group $(P=0.06)$. A cardiovascular death occurred in a patient receiving VKA; two non-cardiovascular deaths were reported (one lung cancer-related death in the EDO group and one bladder cancer-related death in the VKA group). A total of 14 patients (5 in EDO and 8 in VKA) experienced secondary safety outcome. The cumulative incidence of CRNMB events was $3.8 \%$ in the EDO group and $6.1 \%$ in the VKA group $(P=0.03)$. The total anticoagulant therapy discontinuation rate was $4.62 \%(6 / 130)$ in the EDO group and $6.15 \%(8 / 130)$ in the VKA group $(P=0.06)$ (Table 2).

\section{Discussion}

Real-world evidences on clinical performance of non-vitamin $\mathrm{K}$ antagonist oral anticoagulant (NOACs) use in AF patients scheduled for DCC are relevant to address current unmet medical needs and to better define the specific role of such agents in this clinical setting. NOACs offer several potential advantages over VKAs in AF patients undergoing cardioversion, removing the need for routine laboratory monitoring and heparin bridging therapy and reducing time to procedure [18]. The present study is the first real-world experience

Table 1 Baseline characteristics of the study population before and after propensity matching

\begin{tabular}{|c|c|c|c|c|c|c|}
\hline \multirow[t]{2}{*}{ Variable } & \multicolumn{3}{|c|}{ Before propensity score matching } & \multicolumn{3}{|c|}{ After propensity score matching } \\
\hline & $\operatorname{EDO}(n=230)$ & VKA $(n=210)$ & $P$ value & $\operatorname{EDO}(n=130)$ & $\operatorname{VKA}(n=130)$ & $P$ value \\
\hline Age (years) & $61.1 \pm 10.4$ & $73.9 \pm 6.4$ & $<0.001$ & $62.3 \pm 10.2$ & $63.1 \pm 10.3$ & 0.73 \\
\hline Female $(\%)$ & 46.4 & 42.1 & 0.58 & 42.5 & 41.8 & 0.71 \\
\hline BMI $\left(\mathrm{kg} / \mathrm{m}^{2}\right)$ & $27.8 \pm 4.9$ & $29.8 \pm 6.1$ & 0.82 & $26.9 \pm 7.3$ & $26.8 \pm 7.2$ & 0.77 \\
\hline Hypertension (\%) & 49.5 & 59.2 & 0.001 & 54.2 & 56.1 & 0.57 \\
\hline CHA2DS2VASc score & $2.5 \pm 1.7$ & $3.3 \pm 1.3$ & 0.02 & $2.1 \pm 0.6$ & $2.2 \pm 0.4$ & 0.55 \\
\hline HAS-BLED score & $2.2 \pm 1.1$ & $3.2 \pm 1.0$ & 0.001 & $2.05 \pm 1.1$ & $2.04 \pm 1.5$ & 0.55 \\
\hline Diabetes mellitus (\%) & 15 & 22 & 0.06 & 14 & 13 & 0.4 \\
\hline Heart failure $(\%)$ & 17.7 & 27.1 & 0.001 & 21.6 & 22.3 & 0.8 \\
\hline Prior stroke/TIA (\%) & 26.9 & 36.4 & 0.001 & 27.8 & 28.3 & 0.6 \\
\hline Prior MI (\%) & 8.2 & 13.1 & 0.02 & 5.6 & 6.2 & 0.7 \\
\hline $\mathrm{CrCl}(\mathrm{mL} / \mathrm{min})$ & $72.3 \pm 14.1$ & $60.7 \pm 12.9$ & 0.001 & $71.3 \pm 21.1$ & $72.2 \pm 21.2$ & 0.7 \\
\hline Left atrial diameter (mm) & $44.3 \pm 6.7$ & $46.8 \pm 5.7$ & 0.8 & $46.2 \pm 5.3$ & $47.1 \pm 5.7$ & 0.8 \\
\hline LAVI $\left(\mathrm{mL} / \mathrm{m}^{2}\right)$ & $32.3 \pm 1.1$ & $33.7 \pm 1.5$ & 0.42 & $32.3 \pm 2.4$ & $33.2 \pm 1.2$ & 0.7 \\
\hline $\operatorname{LV}$ EF $(\%)$ & $53.4 \pm 6.5$ & $44.3 \pm 6.1$ & 0.002 & $55.4 \pm 5.2$ & $54.3 \pm 3.9$ & 0.8 \\
\hline TEE performed (\%) & 100 & 100 & & 100 & 100 & \\
\hline
\end{tabular}

Values are expressed as mean \pm SD unless otherwise stated

$B M I$ body mass index, $\mathrm{CrCl}$ creatinine clearance, $E D O$ edoxaban, $L A V I$ indexed left atrial volume, $M I$ myocardial infarction, $S D$ standard deviation, $T I A$ transient ischemic attack, $V K A$ vitamin $\mathrm{K}$ antagonist, $L V E F$ left ventricle ejection fraction, $T E E$ transesophageal echocardiogram 
Table 2 Efficacy and safety endpoints and therapy discontinuation rate in the EDO and VKA groups

Variable

\begin{tabular}{llll}
\cline { 2 - 4 } & $\begin{array}{l}\text { EDO } \\
(n=130)\end{array}$ & $\begin{array}{l}\text { VKA } \\
(n=130)\end{array}$ & $P$ value \\
\hline Stroke/TIA/SE & $1.54 \%$ & $2.31 \%$ & 0.9 \\
Major bleedings & $1.54 \%$ & $3.08 \%$ & 0.4 \\
All-cause death & $0.77 \%$ & $1.54 \%$ & 0.06 \\
CRNMB & $3.8 \%$ & $6.1 \%$ & 0.03 \\
Therapy discontinuation rate & $4.62 \%$ & $6.15 \%$ & 0.06 \\
\hline
\end{tabular}

TIA transient ischemic attack, $S E$ systemic embolism, $C R N M B$ clinically non-relevant non-major bleedings

investigating the safety and effectiveness of newly initiated anticoagulation with edoxaban versus well-controlled VKA therapy in patients with non-valvular AF scheduled for TEEguided DCC. Our results did not show a statistically significant difference in the cumulative incidence of both major bleedings and thromboembolic events among AF patients who underwent DCC on edoxaban vs VKA. Our data confirm the results of the ENSURE AF study [16], which showed in 2199 AF patients undergoing DCC a similar cumulative incidence for the composite net clinical benefit outcome of stroke, systemic embolic event, transient ischemic attack, myocardial infarction, cardiovascular mortality, and major bleeding events between edoxaban group vs enoxaparin-VKA group. Moreover, our study showed a low incidence of left atrial thrombus revealed by TEE in AF patients on edoxaban therapy $(0.7 \%)$, similar to that observed in the VKA study group $(0.7 \%)$, but lower than those reported by ENSURE-AF prospective randomized study [16], in which the authors identified left atrial appendage thrombus by TEE in 47 patients $(8 \%)$ in the edoxaban group and in 42 patients $(7.1 \%)$ in the enoxaparin-warfarin group. The low left atrial thrombus incidence reported by the present study was similar to those reported by previous real-world studies analyzing dabigatran $(0.6 \%)$ and apixaban $(0.5 \%)$ clinical performances in AF patients undergoing DCC $[11,13]$. These results may be explained by the different clinical characteristics of our study population compared with those of previous randomized clinical trials (RCTs); our study population showed that mean $\mathrm{CHA}_{2} \mathrm{DS}_{2}$-VASc score values were lower ( $2.1 \pm 0.6$ in EDO group vs $2.2 \pm 0.4$ in VKA group) than those (([2-6 (SD 1.4)) reported by Ensure [16], and similar to values reported by other previous real-world experiences $[11,13]$. The presence of left atrial appendage thrombus at TEE in two patients with thromboembolic risk $\left(\mathrm{CHA}_{2} \mathrm{DS}_{2}\right.$-VASc score $\left.\geq 4\right)$ and moderate renal impairment (GFR $<40 \mathrm{~mL} / \mathrm{min}$ ) supports the hypothesis that these factors might be considered predictors of left atrial thrombus in the real-world setting $[11,13,19,20]$.

\section{Limitations}

The present is a single-centre observational study mainly including low-risk patients with no history of stroke or major bleeding; this is because we usually propose in our clinical practice the rhythm control strategy at the first episode of persistent AF. Our results should be verified in AF patients at moderate-high risk of stroke/major bleeding. Moreover, we compared two groups with several different clinical characteristics; however, the propensity score matching has been used to account the differences. Having a sample size determined by propensity score matching and comparing two proportions with an equivalent test, we decided to set a type I error at $5 \%$ and a non-inferiority margin at $4 \%$. Therefore, in the case of cumulative incidence of major bleedings $(1.54 \%$ in EDO group, $3.08 \%$ in VKA group), we have a power of $62 \%$; in the case of cumulative incidence of thromboembolic events (1.54\% in EDO group and $2.31 \%$ in VKA group), we have a power of $69 \%$. The observation and reporting of adverse effects are usually more accurate and careful in randomized controlled trials than in clinical practice; thus, further studies with wider sizes would be required to detect any differences, if present, in thromboembolic and hemorrhagic events between patients receiving edoxaban and undergoing electrical cardioversion for $\mathrm{AF}$ and those on continuous VKA, given the low incidence of thromboembolic and hemorrhagic events with either strategy.

\section{Conclusion}

There are no real-world data on safety and effectiveness of edoxaban in patients undergoing TEE-guided direct current cardioversion. Our study provides the evidence of a safe and effective use of edoxaban in this clinical setting, justified by no significant difference in major bleedings and thromboembolic events between edoxaban and well-controlled VKA treatments.

Acknowledgments The authors declare that they have no known competing financial interests or personal relationships that could have appeared to influence the work reported in this paper.

Funding Open access funding provided by Università degli Studi della Campania Luigi Vanvitelli within the CRUI-CARE Agreement.

\section{Compliance with Ethical Standards}

Conflict of Interest The authors declare that they have no conflict of interest.

Ethical Approval All procedures performed in studies involving human participants were in accordance with the ethical standards of the institutional and/or national research committee and with the 1964 Helsinki declaration and its later amendments or comparable ethical standards. 
Informed consent was obtained from all individual participants included in the study.

Open Access This article is licensed under a Creative Commons Attribution 4.0 International License, which permits use, sharing, adaptation, distribution and reproduction in any medium or format, as long as you give appropriate credit to the original author(s) and the source, provide a link to the Creative Commons licence, and indicate if changes were made. The images or other third party material in this article are included in the article's Creative Commons licence, unless indicated otherwise in a credit line to the material. If material is not included in the article's Creative Commons licence and your intended use is not permitted by statutory regulation or exceeds the permitted use, you will need to obtain permission directly from the copyright holder. To view a copy of this licence, visit http://creativecommons.org/licenses/by/4.0/.

\section{References}

1. Camm AJ, Kirchhof P, Lip GY, et al. Guidelines for the management of atrial fibrillation: the task force for the management of atrial fibrillation of the European Society of Cardiology (ESC). Eur Heart J. 2010;31(19):2369-429.

2. January CT, Wann LS, Alpert JS, Calkins H, Cigarroa JE, Cleveland JC Jr, et al. AHA/ACC/ HRS guideline for the management of patients with atrial fibrillation: a report of the American College of Cardiology/American Heart Association task force on practice guidelines and the Heart Rhythm Society. Circulation. 2014;130(23):e199-267.

3. Russo V, Bianchi V, Cavallaro C, et al. Efficacy and safety of dabigatran in a "real-life" population at high thromboembolic and hemorrhagic risk: data from MonaldiCare registry. Eur Rev Med Pharmacol Sci. 2015;19(20):3961-7.

4. Russo V, Rago A, Proietti R, di Meo F, Antonio Papa A, Calabrò P, et al. Efficacy and safety of the target-specific oral anticoagulants for stroke prevention in atrial fibrillation: the real-life evidence. Ther Adv Drug Saf. 2017;8(2):67-75.

5. Russo V, Rago A, D'Onofrio A, et al. The clinical performance of dabigatran in the Italian real-life experience. J Cardiovasc Med (Hagerstown). 2017;18(11):922-3.

6. Russo V, Rago A, Papa AA, Meo F, Attena E, Golino P, et al. Use of non-vitamin $\mathrm{K}$ antagonist oral anticoagulants in atrial fibrillation patients with malignancy: clinical practice experience in a single institution and literature review. SeminThrombHemost. 2017;44: $370-6$.

7. Russo V, Attena E, Mazzone C, Esposito F, Parisi V, Bancone C, et al. Nonvitamin $\mathrm{K}$ antagonist oral anticoagulants use in patients with atrial fibrillation and bioprosthetic heart valves/prior surgical valve repair: a multicenter clinical practice experience. Semin Thromb Hemost. 2018;44:364-9.

8. Russo V, Carbone A, Attena E, Rago A, Mazzone C, Proietti R, et al. Clinical benefit of direct oral anticoagulants versus vitamin $\mathrm{K}$ antagonists in patients with atrial fibrillation and bioprosthetic heart valves. Clin Ther. 2019;41(12):2549-57.
9. Russo V, Attena E, Mazzone C, Melillo E, Rago A, Galasso G, et al. Real-life performance of edoxaban in elderly patients with atrial fibrillation: a multicenter propensity score-matched cohort study. Clin Ther. 2019;41(8):1598-604.

10. Ezekowitz MD, Pollack CV Jr, Halperin JL, et al. Apixaban compared to heparin/vitamin $\mathrm{K}$ antagonist in patients with atrial fibrillation scheduled for cardioversion: the EMANATE trial. Eur Heart J. 2018;39(32):2959-71.

11. Russo V, Rago A, Papa AA, D’Onofrio A, Golino P, Nigro G. Efficacy and safety of dabigatran in patients with atrial fibrillation scheduled for transoesophageal echocardiogram-guided direct electrical current cardioversion: a prospective propensity score-matched cohort study. J Thromb Thrombolysis. 2017;45:206-12.

12. Camm AJ, Turpie AGG, Hess S, et al. XANTUS Investigators. Outcomes after catheter ablation and cardioversion in patients with non-valvulara trial fibrillation: results from the prospective, observational XANTUS study. Europace. 2018;20(6):e87-95.

13. Rago A, Papa AA, Cassese A, Arena G, Magliocca MCG, D'Onofrio A, et al. Clinical performance of apixaban vs. vitamin $\mathrm{K}$ antagonists in patients with atrial fibrillation undergoing direct electrical current cardioversion: a prospective propensity scorematched cohort study. Am J Cardiovasc Drugs. 2019;19(4):421-7.

14. Kirchhof P, Benussi S, Kotecha D, Ahlsson A, Atar D, Casadei B, et al. ESC guidelines for the management of atrial fibrillation developed in collaboration with EACTS. Eur Heart J. 2016;37(38): 2893-962.

15. Steffel J, Verhamme P, Potpara TS, et al. The 2018 European Heart Rhythm Association Practical Guide on the use of non-vitamin K antagonist oral anticoagulants in patients with atrial fibrillation. Eur Heart J. ISSN 0195-668X. 2018;39:1330-93.

16. Goette A, Merino JL, Ezekowitz MD, ENSURE-AF investigators, et al. Edoxaban versus enoxaparin-warfarin in patients undergoing cardioversion of atrial fibrillation (ENSURE-AF): a randomised, open-label, phase 3b trial. Lancet. 2016;388(10055):1995-2003 Erratum in: Lancet. 2016 Oct 22;388(10055):1984.

17. Kaatz S, Ahmad D, Spyropoulos AC, Schulman S, the Subcommittee on Control of Anticoagulation. Definition of clinically relevant non-major bleeding in studies of anticoagulants in atrial fibrillation and venous thromboembolic disease in nonsurgical patients: communication from the SSC of the ISTH. J Thromb Haemost. 2015 Nov;13(11):2119-2.

18. Coppola G, Manno G, Mignano A, et al. Management of direct oral anticoagulants in patients with atrial fibrillation undergoing cardioversion. Medicina. 2019;55:660.

19. Stabile G, Russo V, Rapacciuolo A, de Divitiis M, de Simone A, Solimene F, et al. Transesophageal echocardiograpy in patients with persistent atrial fibrillation undergoing electrical cardioversion on new oral anticoagulants: a multi center registry. Int J Cardiol. 2015;184:283-4.

20. Bertaglia E, Anselmino M, Zorzi A, Russo V, Toso E, Peruzza F, et al. NOACs and atrial fibrillation: incidence and predictors of left atrial thrombus in the real world. Int J Cardiol. 2017;249:179-83.

Publisher's Note Springer Nature remains neutral with regard to jurisdictional claims in published maps and institutional affiliations. 